\title{
Emission on the low voltage grid: measurements in an urban area
}

\author{
A. Gil-de-Castro ${ }^{1}$, S.K. Rönnberg ${ }^{2}$, M.H.J. Bollen ${ }^{2}$, M. Wahlberg $^{23}$ and A. Moreno-Munoz ${ }^{1}$ \\ ${ }^{1}$ Departamento A.C., Electrónica y T.E. \\ Escuela Politécnica Superior, University of Cordoba \\ Campus de Rabanales, E-14071 Córdoba (Spain) \\ Phone:+0034 957 212533, e-mail: agil@uco.es \\ ${ }^{2}$ Luleå University of Technology, 93187 Skellefteå, Sweden \\ ${ }^{3}$ Skellefteå Kraft, Skellefteå, Sweden
}

\begin{abstract}
This paper presents the results of a set of measurements to study changes in voltage harmonic emission in LV grid due to the introduction of energy-efficient lamps. The paper involves measurements in an urban area performed in the north of Sweden. Measurements of voltage harmonic were performed before and after replacing all existing (mainly incandescent) lamps with LED lamps in all 12 houses belonging to the same urban area. The total power of all lamps was reduced from approximately $2300 \mathrm{~W}$ to $450 \mathrm{~W}$, per house. All new lamps were low-power-factor lamps. In this paper it has only been shown the results for two individual houses over the twelve that changed their lamps. Those results are then compared with the standard EN 50160 in order to foresee the voltage impact after changing to LED lamps.
\end{abstract}

\section{Key words}

Compact Fluorescent Lamps (CFLs), EN 50160, LED lamps, Power quality, Voltage Distortion.

\section{Introduction}

For the purpose of reducing electrical energy consumption there is an upcoming shift from the traditional incandescent lamps to more energy efficient types of lighting [1]. The most common replacements for incandescent lamps are compact fluorescent lamps (CFL). The next stage will be the replacement by lamps based on light-emitting diodes (LED's). This ongoing replacement has raised the concern among other by the network operators of a possible adverse effect on the power grid as a resistive load is replaced with an electronic one. The main concern is the distortion of the current and voltage waveform [2].

Maintaining a satisfactory power quality is a responsibility that concerns both the electric equipment supplier and the electricity user. Standard EN 50160 [3] indicates the main voltage parameters and their permissible deviation range under normal operating conditions.

This concern has been the reason for starting a set of measurements to quantify the impact of the replacement on the harmonic distortion. Up to now, the current harmonic emission from those measurements has been studied in [4], [5], and [6]. In [4] it was shown that despite the low power factor of the individual lamps, no change in current emission due to the lamps has been observed, neither for any of the two individual houses nor the distribution transformer supplying all 12 houses. Moreover, the paper shows the variations of the most important current harmonics with time and relevant spectra before and after the replacement. Regarding [5], it was shown small changes in current distortion for a hotel, before and after the replacement; showing increases as well as decreases and overall no obvious impact was visible after the replacement of incandescent lamps by energy savings lamps. Finally, [6] is focused in studying changes in current harmonic emission from domestic equipment due to the introduction of energy-efficient lamps. The results from the experiments are compared with standards, with a mathematical aggregation model and with results from field experiments in order to give recommendations to different stakeholders. One of the conclusions of the paper indicates that current emission levels at higher frequencies might increase due to the large-scale introduction of such lamps.

The study presented in this paper is completely based on field measurements. Different PQ monitors (PX5, Elspec, and Hioki) were used to obtain the harmonic distortion and several other parameters at different points. The experiment has been conducted in a way in which voltage harmonics of an installation are calculated from the measured voltage waveforms before and after the replacement of all incandescent lamps by energy-saving lamps. All new lamps were low-power-factor lamps. After the description of the experiments, the paper continues with a presentation of the results.

The rest of the paper is organized as follows. Section 2 reviews the previous literature on this topic; Section 3 of this paper gives details of the measurements that were performed. The results of the measurements are presented in Section 4. Finally Section 5 presents the conclusions. 


\section{Background}

Regarding standards, IEC 61000-3-6 [7] contains the allowable levels for low-, medium-, high- and ultrahighvoltage systems. In this case we consider low-voltage limits.

Table I. - Harmonic voltage limits in LV and MV power systems according to IEC 61000-3-6 [7]

\begin{tabular}{|l|l|l|l|l|l|}
\hline \multicolumn{2}{|l|}{$\begin{array}{l}\text { Odd harmonics non- } \\
\text { multiple of 3 }\end{array}$} & \multicolumn{2}{|l|}{$\begin{array}{l}\text { Odd harmonics } \\
\text { multiple of 3 }\end{array}$} & \multicolumn{2}{l|}{ Even harmonics } \\
\hline $\mathrm{h}$ & Limit (\%) & $\mathrm{h}$ & $\begin{array}{l}\text { Limit } \\
(\%)\end{array}$ & $\mathrm{h}$ & Limit (\%) \\
\hline 5 & 6 & 3 & 5 & 2 & 2 \\
\hline 7 & 5 & 9 & 1.5 & 4 & 1 \\
\hline 11 & 3.5 & 15 & 0.3 & 6 & 0.5 \\
\hline 13 & 3 & 21 & 0.2 & 8 & 0.5 \\
\hline 17 & 2 & $>21$ & 0.2 & 10 & 0.5 \\
\hline 19 & 1.5 & & & 12 & 0.2 \\
\hline 23 & 1.5 & & & $>12$ & 0.2 \\
\hline 25 & 1.5 & & & & \\
\hline$>25$ & $\begin{array}{l}0.2+1.3 *(25 \\
/ \mathrm{h})\end{array}$ & & & & \\
& & & & & \\
\end{tabular}

On the other hand, the European Standard EN 50160 [3] on voltage characteristics of electricity supplied by public distribution networks defines specific limits for (almost all) the continuous parameter variations (including power frequency variations; supply voltage variations; rapid voltage changes; supply voltage unbalance; and harmonic voltages among others) while only supplies indicative values for the voltage events (according to interruptions; voltage dips; voltage swells; and transient overvoltages). Moreover, and regarding harmonic distortion, the standard EN 50160 [3] states that the voltage total harmonic distortion should not exceed $8 \%$ for LV system and $5 \%$ for MV system. In Table II is represented the voltage harmonic level according to the standard.

Table II. - Harmonic voltage limits in LV and MV power systems according to EN 50160 [3]

\begin{tabular}{|l|l|l|l|l|l|}
\hline $\begin{array}{l}\text { Odd harmonics } \\
\text { non-multiple of } 3\end{array}$ & \multicolumn{2}{l|}{$\begin{array}{l}\text { Odd harmonics } \\
\text { multiple of 3 }\end{array}$} & \multicolumn{2}{l|}{ Even harmonics } \\
\hline $\mathrm{h}$ & $\begin{array}{l}\text { Limit } \\
(\%)\end{array}$ & $\mathrm{h}$ & $\begin{array}{l}\text { Limit } \\
(\%)\end{array}$ & $\mathrm{h}$ & $\begin{array}{l}\text { Limit } \\
(\%)\end{array}$ \\
\hline 5 & 6 & 3 & 5 & 2 & 2 \\
\hline 7 & 5 & 9 & 1.5 & 4 & 1 \\
\hline 11 & 3.5 & 15 & 0.5 & $6<\mathrm{h}<24$ & 0.5 \\
\hline 13 & 3 & 21 & 0.5 & & \\
\hline 17 & 2 & & & & \\
\hline $19<\mathrm{h}<25$ & 1.5 & & & & \\
\hline
\end{tabular}

\section{Methodology}

The measurements presented in this paper, were performed in an urban area, part of the municipality of Skellefteå at $65^{\circ} \mathrm{N}$ in the North of Sweden. More results apart from this paper can be found in [4].

A total of 12 households, all supplied from the same distribution transformer, have replaced their incandescent lamps with LED lamps, reducing the installed active power with about $1900 \mathrm{~W}$ each. The maximum decrease of active power due to the replacement of the lamps for the twelve houses together would then be $23 \mathrm{~kW}$.

10 houses over the total of them ( 12 houses) have district heating and the other two have electrical heating. Two houses (with district heating) labeled as T40 and T44 were selected and a power quality instrument was connected at the delivery point of every house and was used to record the voltage harmonic distortion and several other parameters with 10-minute interval. The measurements were performed during the period 201109-12 to 2011-10-13, involving three different periods, before, during and after the replacement. This paper only presents data before and after the replacement.

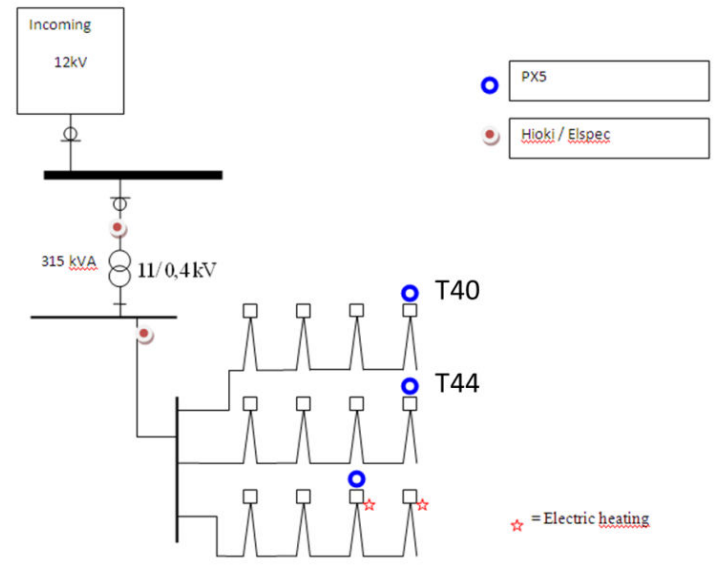

Fig. 1. Supply to the 12 houses and location of the measurement instruments.

The two analyzed houses have two inhabitants each, and the lamps were switched on from approximately 6 to 8 during the morning and from 18 to 23 in the afternoon. This leads us to study the voltage variations over those hours without considering the rest of the hours when it is unlikely that the lamps were switched on.

\section{Measurement results}

A voltage harmonic analysis has been done. First of all, the $24 \mathrm{~h}$ VTHD trend was observed before and after the replacement for both locations T40 and T44 (just phase B has been plotted). A perfectly seen weekday (solid line) daily pattern is observed, while at weekend (dashed line) distinguishing between different hours of the day is difficult. Because of the weekdays/weekend differences, and because of the high VTHD levels from 7.00 in the morning to 16.00 (working hours), we can say that there is a big voltage harmonic dependence from a nearby industry.

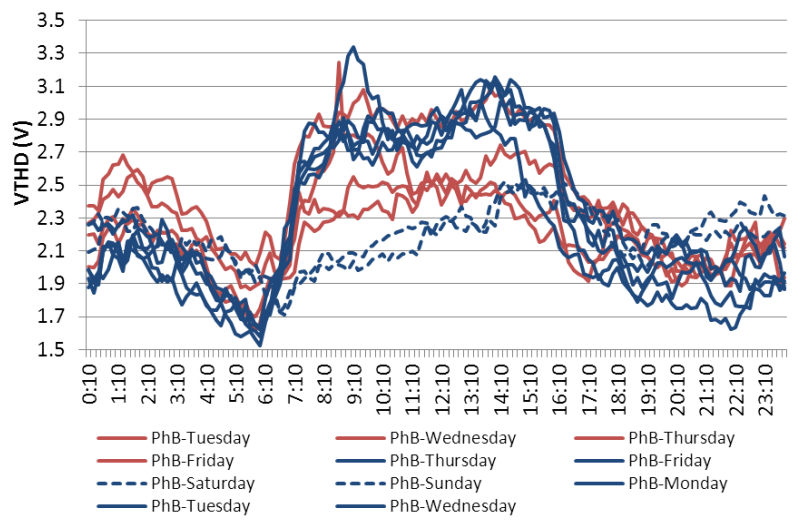

Fig. 2. VTHD (phase B) daily evolution for T40 (blue: before, red: after the replacement). 


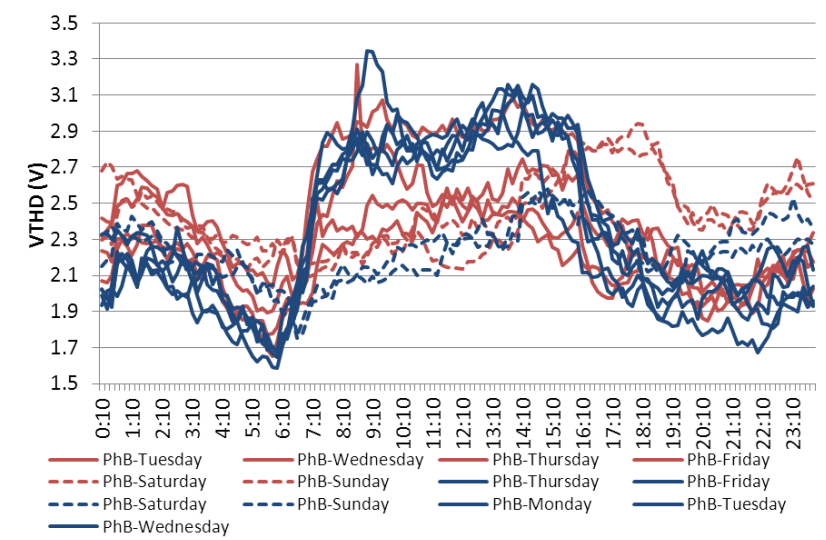

Fig. 3. VTHD (phase B) daily evolution for T44 (blue: before, red: after the replacement).

By knowing this difference between weekdays and weekends, and the impact of the industry, a voltage analysis has been done just considering weekdays. Starting with the rms value, the daily percentage difference of the rms voltage between before and after the replacement states for both houses in weekdays is shown in Fig. 4 and 5 for the three phases. The figures are done by calculating the rms weekday daily average of the voltage before and after the replacement, and calculating then the percentage of change between both states. The positive values mean an increase in the voltage after the replacement.

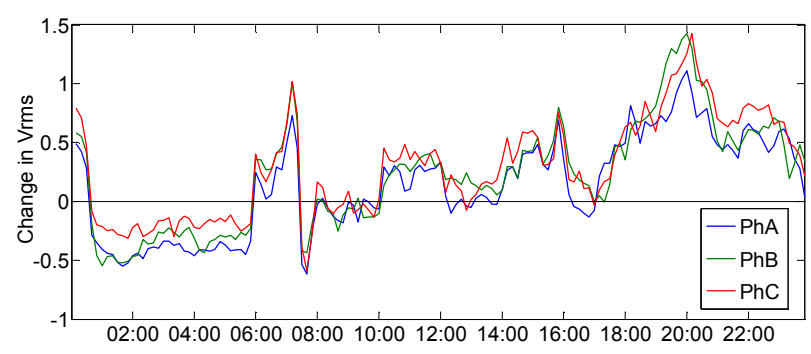

Fig. 4. Change in Vrms from before compared to after the replacement at T40 in weekdays.

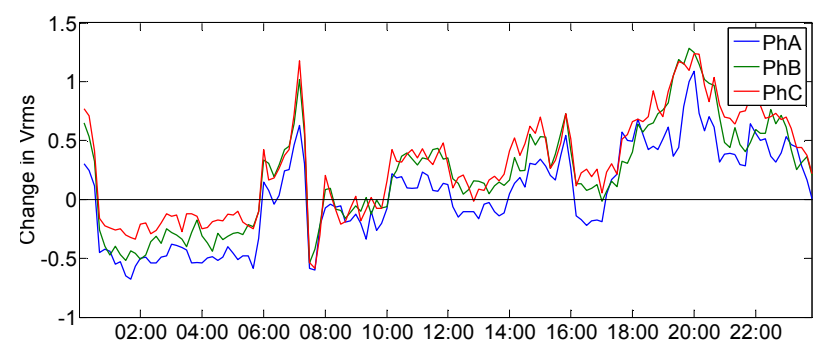

Fig. 5. Change in Vrms from before compared to after the replacement at T44 in weekdays.

As it can be seen from the figures, in the hours when lights must be on (specially from 18:00) there is a small variation after the introduction of LED lamps (it reaches $1.4 \%$ maximum), so overall it can be said that there is not a big impact on voltage levels because the actual voltage levels are low, but it must be into consideration according to the voltage level in each situation. The increase during 6-8 in the morning might be lower compared with the afternoon because of the impact of the nearby industry.

Then, looking at total voltage harmonic distortion (VTHD) in volts, the same plot as it has been done in Fig. 4 and 5 has been done with VTHD. It has been represented the daily difference in percentage from before the replacement compared to after the replacement.

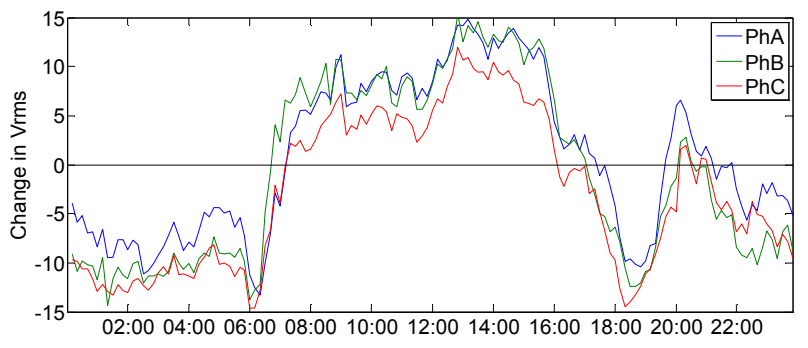

Fig. 6. Change in daily VTHD from before compared to after the replacement for the three phases in weekdays (T40).

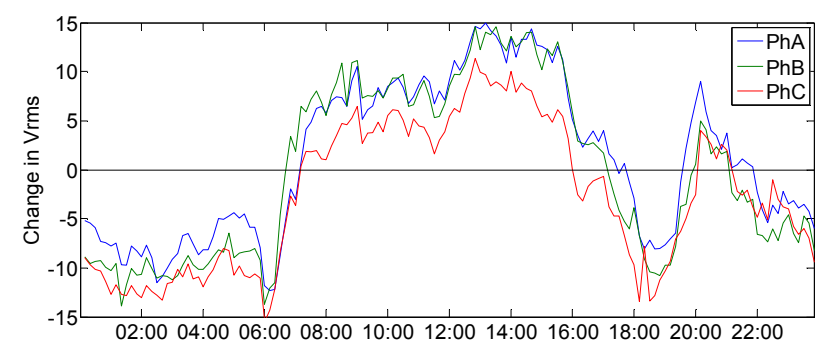

Fig. 7. Change in daily VTHD from before compared to after the replacement for the three phases in weekdays (T44).

It is important to point that the increase given from 7:00 to 16:00 mainly corresponds with the industry; therefore, lamps are not responsible for that. Apart from that, considering the hours when lights must be on (especially afternoon), the THD mostly decreases after the replacement, although a small percentage (average 5\%). The VTHD change varies between $5 \%$ to $-15 \%$, just considering when people had their lights on. There is an increase corresponding with hours that people usually do other activities such as cooking or watching TV.

However, in order to see that overall, the voltage emission is still really low, and this $5 \%$ of increase does not impact so much, every phase has been plotted both before and after the replacement, and shown in Fig. 8 and 9.

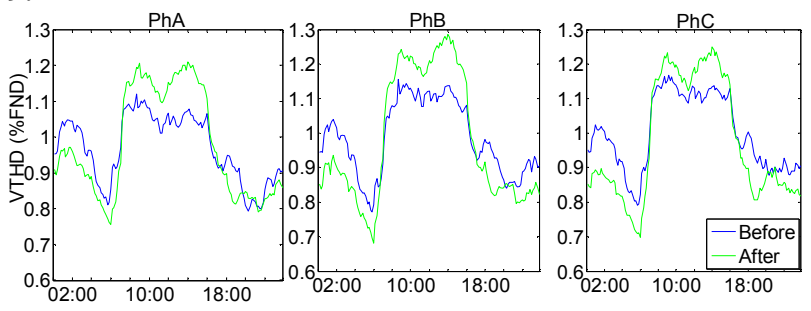

Fig. 8. Daily VTHD (\%) evolution before and after the replacement for the three individual phases in weekdays (T40).

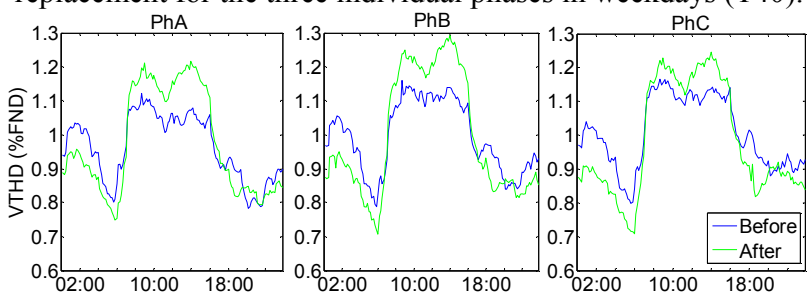

Fig. 9. Daily VTHD (\%) evolution before and after the replacement for the three individual phases in weekdays (T44).

As it can be seen that a small increase or decrease does not influence too much in accomplishing standard, because the VTHD is recommended to be below $8 \%$ and here all the three phases are $1.3 \%$ maximum. Therefore, 
an increase of $10 \%$ makes the VTHD varying between 2.5 $\mathrm{V}$ to $2.7 \mathrm{~V}$, and it is below the standard.

Focusing on low voltage emission, in Fig. 10 and Fig. 11 the $95 \%$ value of the voltage harmonics up to $2 \mathrm{kHz}$ from the whole period (weekdays and weekend) before and after the replacement is presented for the two individual houses. A comparison between the first seven days and the last seven days of the experiment is made. No clear conclusion of an increase or decrease can be made, because some harmonics show an increase and others a decrease.
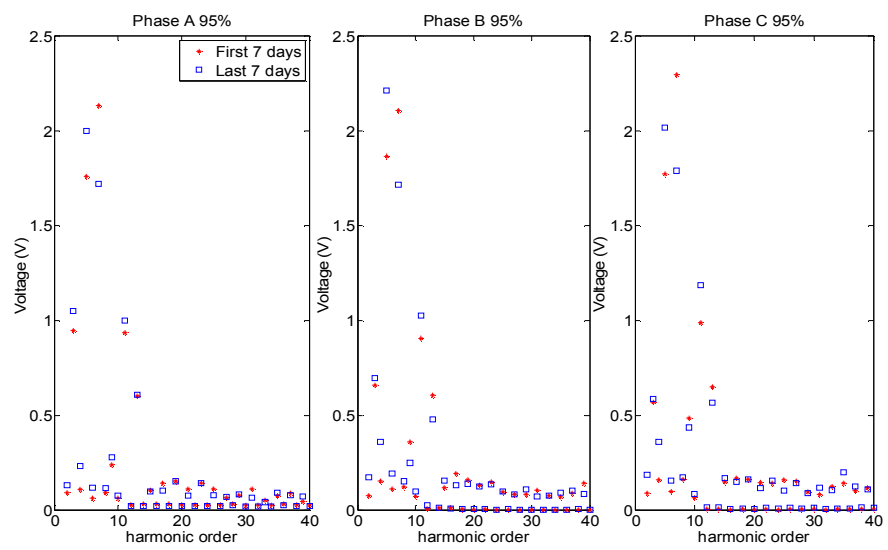

Fig. 10. Change in voltage harmonic spectrum $95 \%$ value before and after change (T40).

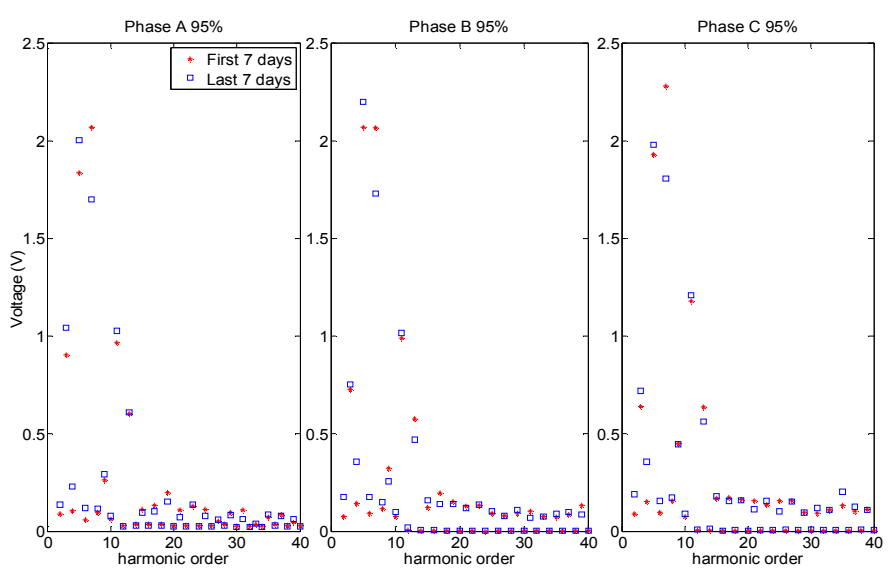

Fig. 11. Change in voltage harmonic spectrum $95 \%$ value before and after change (T44).

In order to avoid possible trends, a weekday daily 95 percentile was then performed. In this case the 95 percentile of each harmonic order, in the same hour but from different weekdays was calculated. Then, it is shown the 95 percentile from the whole day, getting the 95 percentile for all the orders.

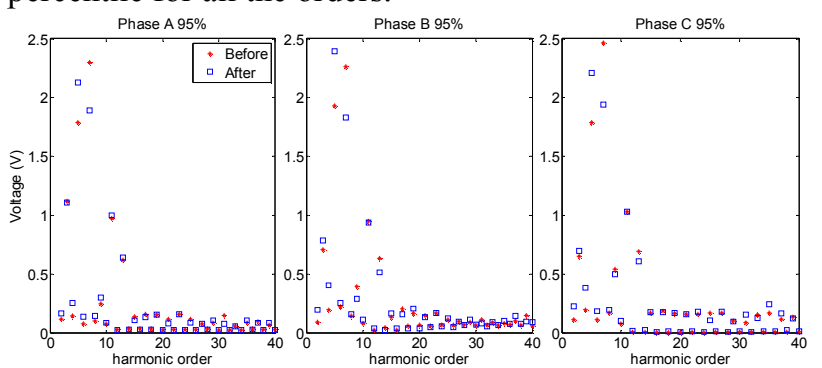

Fig. 12. 95 percentile weekday daily voltage spectrum (T40).

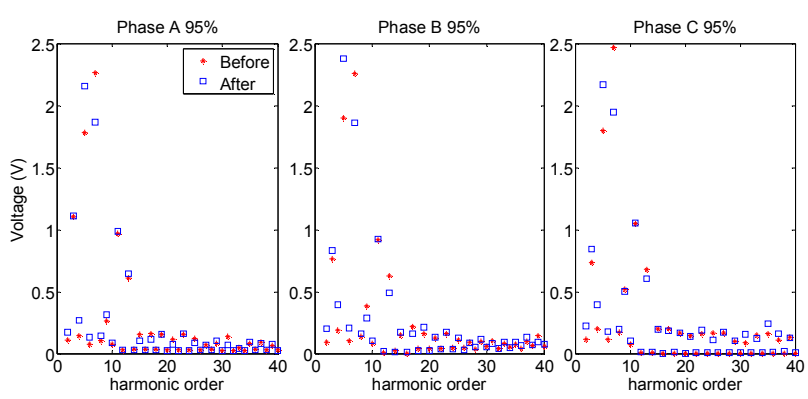

Fig. 13. Average weekday daily voltage spectrum (T44).

In Table III the change in the 95 percentile amplitude for the odd voltage harmonics with two individual houses is presented as an increase (+) or a decrease (-) for the three phases. In Sweden three phases are used in residential buildings and, according to a person familiar with the electrical installations in these houses, the lamps are somewhat equally spread over the three phases.

As can be seen in the table, some harmonics show an increase while others show a decrease when comparing before and after states. More often than not, the level of harmonic both increases and decreases seen over the three different phases. An increase in all phases for the two houses is seen in low orders as $3^{\text {rd }}, 5^{\text {th }}$ and $11^{\text {th }}$. A decrease in all three phases is observed for harmonic 7 in both locations. This is the opposite as found in [8], where this order but in the current shows an increase for all the phases and the two houses.

Table III. - Increase (+) or decrease (-) in voltage harmonic magnitude due to replacement of the lamps, with the two individual houses.

\begin{tabular}{|r|r|r|r|r|r|r|}
\hline & \multicolumn{7}{|c|}{ T40 } & \multicolumn{3}{|c|}{ T44 } \\
\hline & PhA & PhB & PhC & PhA & PhB & PhC \\
\hline 3 & & + & + & & + & + \\
\hline 5 & + & + & + & + & + & + \\
\hline 7 & - & - & - & - & - & - \\
\hline 9 & + & - & - & + & - & - \\
\hline 11 & + & - & & + & + & \\
\hline 13 & + & - & - & + & - & - \\
\hline 15 & - & + & + & - & + & - \\
\hline 17 & - & - & - & - & - & - \\
\hline 19 & - & + & + & + & + & + \\
\hline 21 & - & - & & - & + & - \\
\hline 23 & + & + & + & + & + & + \\
\hline 25 & - & + & - & - & + & - \\
\hline 27 & + & + & + & & - & + \\
\hline 29 & + & + & - & + & + & + \\
\hline 31 & - & - & + & - & - & + \\
\hline 33 & - & + & - & - & + & - \\
\hline 35 & + & + & + & + & + & + \\
\hline 37 & - & + & + & - & + & + \\
\hline 39 & + & - & - & + & - & - \\
\hline
\end{tabular}

Regarding standards, the voltage harmonic emission is limited by the EN 50160 [3] with the limits shown in Table II. Fig. 14 and 15 show odd harmonic orders given by the measurements before and after the replacement for both locations T40 and T44, compared with the limits given by the standard. As it can be seen all are below the actual limits. 


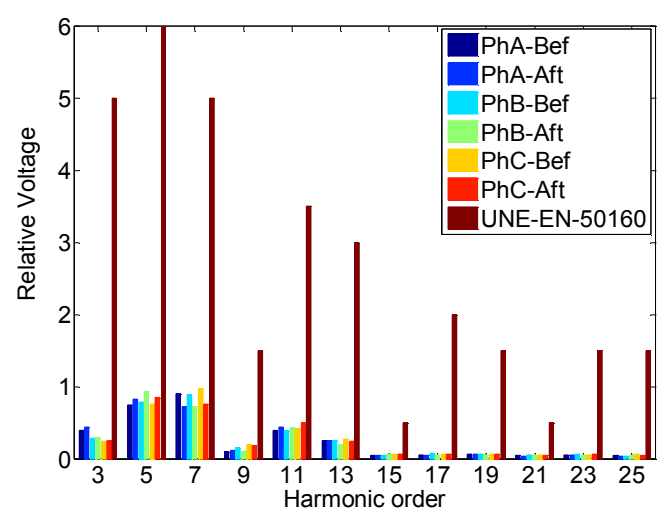

Fig. 14. Relative voltage in T40 compared with EN 50160 [3].

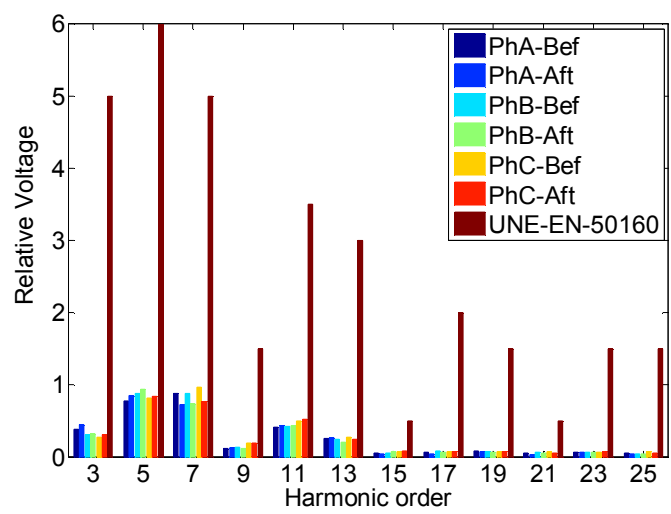

Fig. 15. Relative voltage in T44 compared with EN 50160 [3].

\section{Conclusion}

As far as their current harmonic emission is concerned, energy savings lamps come in two types: those with very high harmonic distortion (ITHD of $80 \%$ of higher) and those with very low harmonic distortion (ITHD of $10 \%$ or lower). Roughly speaking the former group contains small lamps, like CFL and LED lamps, whereas the latter group contains the larger lamps like fluorescent lamps.

In mixed load situations the impact of lamps with high current harmonic distortion remains limited because of their small contribution to the total current. For the domestic installation, under highly controlled conditions, an increase in current emission was visible when all incandescent lamps were replaced. However for the field measurements, where the conditions could not be controlled, no difference was visible.

Not only current but also voltage impact after the replacement must be considered. The first seen aspect is the strong voltage harmonic dependence from a nearby industry, showing a different voltage harmonic pattern between weekdays and weekend. Regarding rms voltage variations after the replacement, the daily percentage difference of the rms voltage shows that there is a small variation after the introduction of LED lamps varying between $1.4 \%$ to $-0.6 \%$ (considering the hours when lights must be on in the houses).

The voltage harmonic emission is another topic to be studied when replacing lamp technology to LED lamps. This paper shows the daily VTHD variation after the replacement. Results show that the VTHD varies between $5 \%$ to $-15 \%$, just considering when people had their lights on (morning and afternoon periods). So, generally it is seen a decrease in the THD after the replacement.
However, after analysing the VTHD in percentage of the fundamental it could be seen that $0.9 \%$ is the normal value when the lamps were on, under the recommended $8 \%$. Therefore, the worst case (an increase of $5 \%$ ) would not impact so much the VTHD for those locations.

Finally, the paper shows a comparison of every individual harmonic order according to the standard EN 50160 [3], showing that no important differences can be seen when comparing before and after the replacement for both houses. In fact they represent a small percentage of those limits.

\section{Acknowledgement}

This research is partially supported by FederInnterconecta project TIGRIS under contract no. 12013095 and by Feder-Innterconecta project PV-On Time under contract no. 12013096.

Part of this work has also been supported financially by Skellefteå Kraft, and the Swedish Energy Administration.

\section{References}

[1] McKinsey Global Institute, "Lighting the way: Perspectives on the global lighting market study," 2011.

[2] N. R. Watson, "Harmonic assessment of electrical power systems," International Journal of Energy Technology and Policy, vol. 4, pp. 6073, 2006.

[3] CENELEC, "EN 50160: 2010. Voltage characteristics of electricity suppplied by public electricity networks," 2010.

[4] S. Rönnberg, M. Wahlberg, and M. Bollen, "Harmonic emission before and after changing to LED lamps - Field Measurements for an urban area," in Harmonics and Quality of Power (ICHQP), 2012 15th International Conference on, 2012.

[5] S. K. Rönnberg, M. Wahlberg, and M. H. J. Bollen, "Harmonic emission before and after changing to LED and CFL - Part II: Field measurements for a hotel," in Harmonics and Quality of Power (ICHQP), 2010 14th International Conference on, 2010, pp. 1-6.

[6] A. Gil-De-Castro, S. K. Rönnberg, M. H. J. Bollen, and A. Moreno-Muñoz, "Study on harmonic emission of domestic equipment combined with different types of lighting," International Journal of Electrical Power and Energy Systems, vol. 55, pp. 116-127, 2014.

[7] "IEC/TR 61000-3-6:2008 Electromagnetic compatibility (EMC) - Part 3-6: Limits Assessment of emission limits for the connection of distorting installations to $\mathrm{MV}, \mathrm{HV}$ and EHV power systems ", February 2008.

[8] S. K. Ronnberg, M. Wahlberg, and M. H. J. Bollen, "Harmonic emission before and after changing to LED lamps - Field Measurements for an urban area," in Harmonics and Quality of Power (ICHQP), 2012 15th International Conference on, 2012, pp. 1-6. 